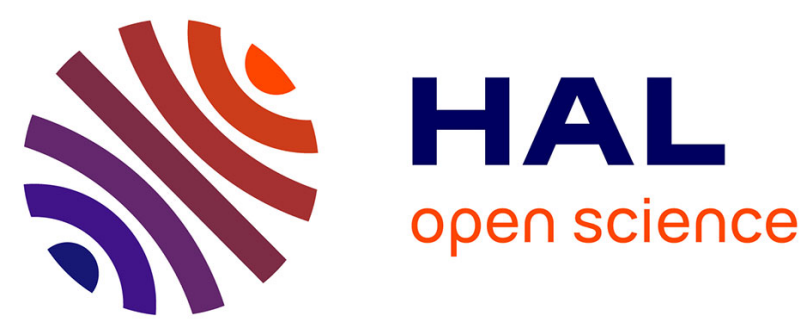

\title{
TRAUMATISME PSYCHIQUE ET ENVIRONNEMENT DÉFAILLANT CHEZ LES INDIVIDUS EN SITUATION DE PRÉCARITÉ SOCIALE
}

\author{
Sophie Fierdepied, Gésine Sturm, Thierry Baubet
}

\section{To cite this version:}

Sophie Fierdepied, Gésine Sturm, Thierry Baubet. TRAUMATISME PSYCHIQUE ET ENVIRONNEMENT DÉFAILLANT CHEZ LES INDIVIDUS EN SITUATION DE PRÉCARITÉ SOCIALE.

Bulletin de psychologie, 2016, 541, pp.3-17. 10.3917/bupsy.541.0003 . halshs-01352757

\section{HAL Id: halshs-01352757 \\ https://shs.hal.science/halshs-01352757}

Submitted on 9 Aug 2016

HAL is a multi-disciplinary open access archive for the deposit and dissemination of scientific research documents, whether they are published or not. The documents may come from teaching and research institutions in France or abroad, or from public or private research centers.
L'archive ouverte pluridisciplinaire HAL, est destinée au dépôt et à la diffusion de documents scientifiques de niveau recherche, publiés ou non, émanant des établissements d'enseignement et de recherche français ou étrangers, des laboratoires publics ou privés. 


\title{
Traumatisme psychique et environnement défaillant chez
}

\author{
les individus en situation de précarité sociale
}

\author{
Fierdepied Sophie *
}

Sturm Gesine **

Baubet Thierry *

\begin{abstract}
Résumé
Cette recherche qualitative tente de comprendre les liens entre trauma, fonctionnements limites et précarité sociale. Les entretiens ont été inspirés de l'Operationalized Psychodynamic Diagnosis (OPD-2). 20 interviews ont été recueillis auprès de 10 sujets. Ils ont été traités grâce à la grounded theory et analysés selon le principe du complémentarisme. Les données récoltées confirment des situations traumatogènes précoces qui reposent autant sur les événements que sur l'inadéquation des réponses environnementales niant la subjectivité des sujets. La logique de survie qui domine leur fonctionnement psychique apparaît dans des modes relationnels empreints de méfiance. La précarité sociale leur offre alors la possibilité de se tenir à l'écart du monde, tout en se positionnant comme sujet.
\end{abstract}

Psychological trauma and failed environment among people in social precarity.

\begin{abstract}
This qualitative research try to understand the relationship between trauma, borderline personality organization et social precarity. The interviews was designed while referring to operationalized Psychodynamic Diagnosis (OPD-2). We made 20 interviews with 10 different persons. They analyzed while using a grounded theory methodology which integrates aspects of Devereux complementarist approach. Data show high rates of potentially traumatogenic situations experienced by the interviewees, confronting them to traumatic events and lacking or inadequate support, and a denial of their subjectivity. The logic of survival that dominates their mental functioning appears in type of relationship marked by mistrust. Sometimes they also experience social precarity as a possibility to stand apart from the world, while positioning themselves as subject.
\end{abstract}

INTRODUCTION

\footnotetext{
* E-A 4403 (UTRPP) Université Paris 13, Sorbonne Paris Cité, Inserm U669, Villetaneuse.

** Université Toulouse-Jean Jaurès, Laboratoire cliniques pathologique et interculturelle (LCPI), UFR de psychologie, Département clinique du sujet, Toulouse.

Correspondance : Sophie Fierdepied, Université Paris 13, Sorbonne Paris Cité, Inserm U669, 93430,

Villetaneuse, France.

< fierdepied@hotmail.com>
} 
Cet article rend compte d'un point spécifique de notre recherche doctorale. De manière générale, au travers d'une méthode qualitative, il s'agit de comprendre les liens entre trauma, précarité sociale et fonctionnements psychiques particuliers. La fréquence des traumas dans les parcours de vie des sujets en situation de précarité sociale ressort dans la clinique. En outre, des fonctionnements psychiques, qui pourraient rappeler des pathologies limites et narcissiques apparaissent de manière récurrente. Cette recherche tente donc d'examiner différentes situations traumatogènes au regard d'éléments psychiques communs à des sujets qui évoluent dans un contexte de précarité sociale.

Elle met en lumière la logique de survie dans laquelle sont engagés psychiquement ces sujets. Cette logique originelle, issue des premières relations entre le petit d'Homme, vulnérable, et les êtres dont dépend sa survie, l'oblige, paradoxalement, à tisser une relation de confiance avec ceux dont il est à la merci. Ce nouage relationnel lui permettra de s'épanouir comme sujet et non plus simplement de survivre : il apprendra ainsi à vivre dans ce monde chaotique en l'habillant de sens et en faisant abstraction du danger que représente autrui.

Or, chez les sujets que nous avons rencontrés, des événements de vie dramatiques vécus précocement les ont confrontés, dans le réel, à la violence fondamentale (Bergeret, 1984), rendant insécurisantes les relations à autrui et remettant en question le pacte social. Cette logique de survie prédomine leurs fonctionnements psychiques. La précarité sociale, utilisant la rue comme mode de vie, entre en corrélation avec cette logique.

Une recherche préliminaire (Fierdepied, Sturm, Taïeb, Moro, Baubet, 2012) nous a permis de montrer la dimension traumatogène de l'exclusion sociale. Elle peut avoir une fonction de mise en sens, tout comme elle peut rester absurde, sidérante et répéter un scénario traumatique de rupture et d'abandon. Quelle que soit la manière dont les sujets vont la vivre, elle met en évidence, au-delà d' une simple situation de pauvreté, une précarité des liens à autrui et à la 
société. Elle entre en corrélation avec des difficultés psychiques, comme le montrent de nombreuses études épidémiologiques.

La plupart de ces études soulignent des troubles anxiodépressifs fréquents, des troubles de la personnalité cinq fois plus élevés que dans la population générale (Laporte, Chauvin, 2009). Plus précisément, d'après les études recensées par Kovess-Masfety (2001), 57,6 \% ont une personnalité pathologique, $41 \%$ des troubles de l'humeur, 33,9\% l'usage de substances psycho-actives, $16 \%$ sont psychotiques, et 33,7\% présentent des troubles dépressifs.

L'étude de Fazel, Khosla, Doll et Geddes (2008) est particulièrement intéressante : il s'agit d'une méta-analyse de 29 études réalisées entre 1967 et 2007 sur la santé mentale des personnes sans logement. Elle montre que les troubles de la personnalité y sont importants $(23,1 \%)$ c'est-à-dire bien plus que les psychoses $(12,7 \%)$ et les troubles dépressifs majeurs (11,4 \%). Schiltz, Houbre et Martiny (2007) avancent l'idée que le fonctionnement psychique de nombreux sujets en situation de précarité correspond à la description des états-limites, dont l'étiologie traumatique entraînerait, par la suite, une biographie défavorable. Il serait question d'une boucle rétroactive entre l'organisation de la personnalité et la réaction aux stresseurs extérieurs.

\section{REVUE DE LA LITTÉRATURE}

\section{La notion de trauma}

Connue depuis l'antiquité, la notion de psychotraumatisme a été redécouverte au XIX ${ }^{\mathrm{e}}$ siècle par Oppenheim (Crocq, 2012). C'est une question qui devient alors centrale, au point qu'elle sera à la base de la réflexion qui conduira à la découverte de la psychanalyse. Comme on le sait, Freud explique le trauma comme étiologie des troubles hystériques. Rapidement, il s'en détache, non pas en niant l'existence d'attentats réels (il en recherche l'existence dans les 
récits de ses patients, comme le souligne Françoise Brette (1988)), mais en avançant l'idée qu'une situation de séduction, rencontrant les fantasmes de l'enfant, peut faire traumatisme.

Avec la Première Guerre mondiale, se développe la notion de névrose de guerre ou névrose traumatique. Freud introduit, à partir de 1920, la notion d'effraction du pare-excitation, dans laquelle la dimension économique est primordiale : l'effraction traumatique correspond à un trop plein d'excitation face à laquelle les stratégies défensives habituelles ne suffisent plus à protéger le Moi. Cette définition correspond à la description de la névrose traumatique, définie comme un événement majeur, suscitant l'effroi au travers du surgissement soudain du réel de la mort (Lebigot, 2001).

À partir de 1926, Freud offre une nouvelle théorie de l'angoisse et envisage, alors, le traumatisme en dehors de toute référence à la névrose traumatique. Il est, en effet, ici, question de la perte de l'objet provoquant un sentiment de détresse (Hiflosigkeit). C'est à partir de cette nouvelle théorie, mais, aussi, de la référence, dans L 'homme Moïse (Freud, 1939), aux blessures précoces du Moi, que de nombreux auteurs ont élargi la notion de traumatisme à celle de traumatisme narcissique. Winnicott, Green, Roussillon citent des situations qui confrontent le sujet au sentiment de son anéantissement psychique, provoquant alors une « terreur agonistique » (Roussillon, 1999), une « terreur sans nom » (Bion 1962), une « agonie primitive » (Winnicott, 1969). Elles sont particulièrement toxiques chez un enfant ou un adolescent qui, comme Ferenczi l'a souligné (1932), implique un être dont le développement n'est pas terminé. Pour Bergeret et Reid (1986), il peut s'agir d'un traumatisme infantile majeur ou d'une série de traumatismes cumulés, qui vont rendre impossible l'équilibre narcissique. Pour Green, le traumatisme peut, aussi, être lié à un désinvestissement trop précoce ou trop brutal de la mère, entraînant une désillusion insupportable. Il parle de traumatisme narcissique (Green, 1983). Quand il arrive précocement, le trauma est non représenté et ne laisse de trace que son absence de trace. 
Green (1999) parle, pour cette raison, de traumatisme en creux. Pour Roussillon (1999), il faut considérer comme traumatiques toutes situations où un enfant est soumis, de manière répétée, à une logique paradoxale. Ces situations induisent un traumatisme de non-sens, qui oblige le Moi à s'adapter, quitte à se déformer. Elles donnent lieu à des fonctionnements psychiques particuliers tels que pathologies du self, du narcissisme, psychoses blanches, fonctionnements ou états limites (Brette, 1988). Il s'agit de sujets qui luttent constamment contre des expériences internes de désintégration, de dépersonnalisation, de perte du sens du réel, de la capacité relationnelle, que Winnicott (1969) a présenté comme « crainte de l'effondrement ». Elle induit des stratégies défensives, comme le double retournement, la projection et le clivage.

Si l'impact narcissique est capital, comme nous venons de le voir, le processus de subjectivation est, lui aussi, mis à mal, puisque ces situations traumatiques produisent toujours un déni des éprouvés du sujet, qui, en réalité, est un déni de sa subjectivité. Pour en comprendre l'impact, il faut s'arrêter un instant sur le concept de subjectivation.

\section{Le concept de subjectivation}

Concept nomade, le terme de subjectivation est issu de la philosophie. Bien que repris par Lacan (1966) pour montrer que le sujet se détermine à partir de l'Autre premier, cette notion de subjectivation n'est utilisée que récemment en psychanalyse. Elle fait référence à la capacité de l'appareil psychique à exercer une activité, à l'analyser et, ainsi, à y avoir accès par inflexion, dans le but de la modifier. Il s'agit donc d'un processus qui provoque une transformation, par l'appropriation de son appareil psychique. Pour Wainrib (2006), cette notion signifie, à la fois, « rendre subjectif », c'est-à-dire donner du sens, mais, aussi, « devenir sujet ». Dans son premier sens, la subjectivation est en lien avec la subjectalisation, 
qui consiste à se vivre comme distinct d'autrui et fait référence au processus de séparationindividuation. Dans son second sens, la subjectivation fait référence à un processus d'acquisition, d'apprivoisement par un sujet, de son fonctionnement psychique, participant, ainsi, à sa continuité d'être. Wainrib en propose la définition suivante : la subjectivation est « ce processus, en partie inconscient, par lequel un individu se reconnaît dans sa manière de donner sens au réel, au moyen d'une activité de symbolisation ». Il précise que « ce terme renvoie aussi bien au corps propre, à ses pulsions, qu'à l'environnement » (Wainrib, 2006, p. 22). Ceci implique que le sujet émerge en l'individu, en même temps que sa réalité psychique.

Pour Roussillon (2006), l'appropriation subjective est un impératif narcissique, qui consiste à se vivre comme acteur de ce qui se produit en soi et autour de soi. La subjectivation est, donc, un processus qui se poursuivra tout au long de la vie du sujet et devra tendre vers un Moi unifié, conscient de lui-même comme de ses limites, conscient du monde, de sa capacité à interagir avec lui et de lui donner du sens. La possibilité d'affronter une situation traumatogène tiendrait, également, au bon déroulement du processus de subjectivation.

Bon nombre de pathologies, allant des fonctionnements limites aux psychoses, sont, en réalité, des pathologies de la subjectivation : ce processus ne peut, en effet, avoir lieu sans identification ni reconnaissance, c'est-à-dire sans que l'individu se voie se refléter dans les yeux d'autrui. De plus, certains mécanismes de défense (déni, clivage, identification projective, acting...), appelés par Cahn (2006, p. 11) « mécanismes de désinvestissement », réduiraient les capacités à devenir sujet. Il s'agit, donc, d'un engagement difficile dans ce processus, aussi bien dans la manière d'être, vécu par son environnement que dans la mise en place de défenses psychiques.

\section{PROBLÉMATIQUE ET MÉTHODOLOGIE}


Il s'agit de comprendre la manière dont s'articulent des vécus traumatiques avec des fonctionnements psychiques particuliers, qui s'expriment dans une situation sociale qu'est la précarité. Pour cela, le vécu subjectif des individus, face à leur situation sociale a été analysé ainsi que leur récit de vie. Cette recherche qualitative s'étaye sur plusieurs outils méthodologiques ouverts sur les sciences humaines. En effet, son objet, au carrefour de la psychologie et de la sociologie, nécessite de laisser parler différents champs, au risque, sinon, d'enfermer le chercheur aveuglément dans l'un ou l'autre. La réalisation des entretiens s'est inspirée de l'Operationalized Psychodynamic Diagnosis-2 (OPD Task Force, 2008), outil multi-modèle et multi-axial, utilisé pour le diagnostic psychique. Dix sujets en situation de précarité ont été rencontrés, au cours de vingt entretiens semi-directifs, réalisés dans une structure d'accueil de jour pour personnes en situation de précarité à Bourges (Cher). L'utilisation de la théorie ancrée, issue de la sociologie (plus précisément de l'interactionnisme symbolique), pour leur traitement, a permis de comparer et regrouper de nombreuses données sous forme de thématiques émergentes (catégories conceptuelles) de plus en plus générales. L'analyse clinique de ces catégories s'est appuyée sur la méthode complémentariste de Devereux (1967). Elle permet de faire intervenir des notions issues de plusieurs champs théoriques, comme la sociologie et la philosophie, mais offre, également, différents niveaux de lecture d'un même phénomène.

\section{Entretiens de recherche}

L'élaboration du déroulé de ces entretiens, devait permettre, non seulement, d'étudier le vécu de précarité des sujets, mais aussi, de mettre en lien cette expérience avec un parcours de vie antérieur. L’idée de revoir les sujets lors de plusieurs entretiens, devait faciliter une relation de confiance avec eux permettant de connaître, avec plus de précision, leur parcours 
de vie, mais, surtout, de laisser apparaître une tonalité contre-transférentielle, utile dans la compréhension de leur mode de fonctionnement.

L' OPD-2 présentait un intérêt pour l'élaboration de nos entretiens ; en effet, il s'agit, d'une part, d'un outil multimodèle, fondé, autant sur les classifications psychiatriques, que sur les concepts psychanalytiques, psychodynamiques ou psychosomatiques. Il est, d'autre part, multiaxial : il permet d'étudier le sujet sous différentes dimensions (son mode relationnel, son anamnèse, sa structure psychique, ses conflits, ses mécanismes de défense). Cet outil a montré sa validité comme outil diagnostique, mais, aussi, comme outil de communication entre professionnels de champs différents. Il aide à déterminer les axes thérapeutiques et apporte une véritable fiabilité clinique (Cierpka et coll., 2007).

L'OPD-2 a donc inspiré la construction d'une série de trois entretiens avec chacun des sujets recrutés : le principe d'un processus évolutif d'entretiens, largement inspiré par la technique de Kernberg, allant d'une phase de questions ouvertes à des questions plus fermées a été adopté ainsi que le principe d'exploration déclinés par cet outil s'appuyant sur cinq axes liés au fonctionnement des sujets. Ces axes sont présentés ci-dessous :

1. L'expérience de la maladie et les conditions préalables au traitement : cet axe a été modifié afin de porter sur l'expérience que les sujets avaient de leur situation sociale.

2. Les relations interpersonnelles : cet axe a été conservé par l'interrogation systématique des sujets sur leur rapport aux autres et en prêtant attention aux relations transférentielles. Ces dernières ont été consignées dans un journal de recherche.

3. Les conflits : au cours des entretiens, l'attention a été portée sur l'expression de conflits psychiques et, notamment, sur les mécanismes de défense utilisés.

4. La structure : cet axe permet de déterminer le type de structure psychique présenté par l'OPD-2, au travers de quatre niveaux différents d'intégration. Il a été utilisé pour vérifier le 
niveau de cohérence des récits : hors réalité, peu cohérents, fantasmatiques ou en phase avec la réalité. L'attention a été portée notamment sur la capacité des sujets à critiquer leurs possibles contradictions, les thématiques, pour lesquelles les incohérences pouvaient être les plus fortes et qui nous donnaient des indications sur leur rapport à la réalité.

5. Les désordres psychosomatiques et mentaux : il s'agit d'un croisement entre plusieurs outils de classification (pour cette recherche, psychiatriques et psychanalytique) qui permet la prise en compte des différents symptômes.

L'OPD-2 nous a donc permis de bâtir nos entretiens, en ouvrant, autant que possible, les multiples aspects de la personnalité des sujets. Il a, en outre, éloigné le risque d'influencer le matériel, en occultant certaines dimensions de la personnalité ou bien en insistant trop sur d'autres. De plus, cet outil était un véritable guide, pour que chaque sujet soit interrogé, de manière systématique, sur les mêmes éléments. Dans la première phase de l'entretien (phase ouverte), il était important d'être particulièrement attentif à la présentation des sujets, leur aspect, leurs conduites, leur langage. Les effets contre-transférentiels ont, évidemment, été pris en compte dès ces premiers instants de la rencontre avec chacun des sujets et notés dans le journal de recherche.

La seconde phase de l'entretien a été plus structurée autour de la situation de précarité sociale, les effets psychiques, que le sujet peut en ressentir, la perception qu'il a de lui, des autres, les pensées qui l'animent. Il était question de connaître son histoire individuelle et familiale, en étant vigilant quant aux éléments traumatiques mentionnés spontanément. De manière générale, un soin particulier a été pris de relever les traits de personnalité, les symptômes éventuels, les mécanismes de défense. 
Le deuxième entretien, réalisé quinze jours après le premier, avait, pour objectif, de confirmer ou d'infirmer certains aspects du fonctionnement psychique reconnu lors du premier entretien, de les explorer de manière plus approfondie, tout comme les mécanismes de défense, les relations transférentielles, en prenant appui sur la mise en place d'une relation transférentielle. Cet entretien devait permettre d'aller plus loin dans l'exploration du vécu subjectif du sujet. Enfin, elle devait permettre de revenir et d'approfondir la place des événements de vie marquants dans son vécu actuel.

Le troisième et dernier entretien, six mois plus tard, avait, pour objectif, de pouvoir comparer les évolutions individuelles ayant eu lieu pendant ce laps de temps et de les mettre en relation avec des variables environnementales. Il devait, également, permettre de proposer les premières pistes de réflexion aux sujets.

\section{Traitement des données}

Le traitement des données s'appuie sur la méthode de la théorie ancrée. Son principe est de faire émerger des éléments théorisables, à partir de données issues de la subjectivité des individus exposés à la situation de précarité sociale.

La théorie ancrée s'appuie sur la comparaison continue des données, une comparaison qui intervient à tout moment du processus d'analyse. Elle implique, non pas un processus d'analyse linéaire, mais de perpétuelles allées et venues entre l'analyse et les données. Elle permet d'en dégager les différences et les similitudes, d'en comprendre les liens existant entre elles, leurs logiques, leurs sens cachés dans le matériel pour créer une théorisation (Glaser, Strauss, 1967). Cette méthode, au départ purement inductive, a été, par la suite, développée par Strauss et Corbin (1990) dans le sens d'une interprétation n'excluant pas la référence à la théorie. L'originalité de cette méthode consiste à ouvrir le processus d'interprétation, sans 
réduire les données aux théories existantes préalablement. Elle contraint à la comparaison constante du matériel, tant dans sa globalité que dans ses éléments de détails. Grâce à la théorie ancrée, il a été possible de traiter, organiser et regrouper les données et de formuler six catégories, qui reprennent, sous forme d'une conceptualisation, les thématiques centrales apparaissant dans l'ensemble des 20 entretiens analysés. Ces catégories sont le résultat de l'analyse par la théorie ancrée. Elles sont issues de l'interprétation du matériel présentée sous la forme conceptualisée, comme le précisent Paillé et Mucchielli (2012). Une particularité importante de la démarche d'interprétation réside dans le fait qu'elle inclut une interprétation des dynamiques inconscientes et qu'elle propose, ainsi, l'intégration d'une lecture psychanalytique du matériel dans l'usage de la théorie ancrée (Sturm, Nadig, Moro, 2010).

La question de recherche était d'abord formulée de manière très ouverte, afin de favoriser l'émergence de facteurs, qui pourraient nous instruire sur les liens complexes entre précarité, événements traumatiques et fonctionnement psychique. Fidèle à la perspective de Glaser et Strauss, aucune hypothèse n'a été formulée. En revanche, l'idée de Strauss et Corbin, consistant à formuler des intuitions au niveau de l'interprétation a été adoptée, afin de pouvoir les développer au cours de l'analyse (Strauss, Corbin, 1990).

Les interrogations portaient sur les liens entre une situation sociale particulière et des fonctionnements psychiques, qui, sans être homogènes ni uniformes, montraient des points communs. Enfin, il était question de comprendre comment la précarité sociale était subjectivement vécue par rapport au parcours de vie marqués, bien souvent, par des événements douloureux. La théorie ancrée est apparue comme la méthode qualitative la plus adaptée pour ses capacités à conceptualiser des interprétations de données. De plus, elle favorise un approfondissement des données recueillies, puisqu'elle oblige à tenir compte de tous les éléments en présence, sans en omettre aucun, permettant, ainsi, de découvrir des 
éléments insoupçonnés. La théorie ancrée permet, également, de « laisser parler » le matériel, en en tirant une interprétation la plus fidèle possible du discours des sujets rencontrés, ancrée sur lui. Notre familiarité clinique avec le matériel pouvait nous faire craindre d'orienter involontairement l'analyse des données. Nous ne prétendons pas, pour autant, que notre subjectivité est « extraite » du traitement des données, mais la méthode de la théorie ancrée permettait d'éviter qu'elle limite l'interprétation à notre seul regard de clinicien et notre expérience du terrain. L'intégration d'une réflexion sur notre position et nos réactions contretransférentielles nous ont permis d'approfondir et d'enrichir les interprétations.

\section{Analyse des données traitées}

Quand cela est nécessaire, c'est-à-dire quand les éléments théoriques, issus de la psychologie n'apportent pas d'éclairage suffisant pour aller plus loin dans l'analyse, la méthode complémentarisme de Devereux $(1967,1970,1972)$ a été appliqué. Elle consiste à soumettre les données d'une recherche à des lectures différentes et indépendantes, sous l'angle psychologique, sociologique, anthropologique ou autre. Cette méthode est dite complémentariste, dans le sens où l'utilisation de différents champs théoriques qui se complètent, permet de mieux comprendre la complexité d'une situation donnée. Le complémentarisme peut être défini, comme nous le rappellent Sturm et coll. (2010), comme « une stratégie de triangulation pour les projets de recherche interdisciplinaires, où différentes perspectives sur le matériel de recherche sont combinées ».

\section{RÉSULTATS}

Dix sujets différents ont été interrogés pour cette recherche et vingt entretiens ont été réalisés : trois sujets ont été rencontrés trois fois, quatre sujets deux fois et trois sujets une 
fois. Peu fixés à un endroit, certains avaient quitté la région, d'autres ne se sont pas rendez au rendez-vous fixé.

Ces dix sujets (6 hommes et 4 femmes) sont tous en situation de précarité sociale depuis plusieurs mois, voire plusieurs années. Cinq d'entre eux sont sans abri (Christophe, Albert, Léon vivent dans leurs véhicules) ou sans domicile fixe au moment où nous les rencontrons (Mick et Vanille vivent entre foyers d'urgence et squats). Deux ont un logement instable (Romane est en cours d'expulsion, Martin vit dans un logement insalubre, qu'il fuit la plupart du temps). Enfin, trois autres (Laury, Claire et Anne) sont en appartement, sans risque immédiat de le quitter, stabilisés par une mesure de protection pour Claire et Anne. Deux d'entre eux seulement vivent en couple. Presque tous les sujets (hormis Mick) conservent des liens familiaux, mais qui sont particulièrement distendus.

Dans ces récits de vie, des situations allant de changements catastrophiques, d'événements bouleversants, à de véritables traumas sont repérables. Parfois, il s'agit de trauma unique (Léon et Albert, en lien avec un accident de voiture), de traumas répétés (Claire et Anne autour de violences sexuelles ; Vanille, Martin, Mick, autour de maltraitances). Enfin, trois des sujets ont vécu ce que l'on peut qualifier de changements catastrophiques (Christophe, Romane, Laury). Ces événements ont, pour presque tous les sujets (hormis Albert, dont nous ne savons rien de son parcours infantile et adolescent), la particularité d'avoir fait effraction au cours de leur minorité. Ainsi, les récits de vie qui nous ont été faits, trouvent leur origine dans des événements dramatiques.

Chacun des vingt entretiens a été retranscrit puis découpé en petites séquences. Chacune de ces bribes a donné lieu à un codage très descriptif et chacun de ces codages a été comparé aux autres et regroupé en fonction de leurs similitudes, créant, alors, des catégories de plus en plus 
générales. Grâce au logiciel N'VIVO, version $9.2^{1}$, plus de deux mille trois cents bribes d'entretiens ont été codés, formant un arbre de catégorisation, créant et structurant six catégories finales, servant de base à la partie discussion de cette recherche. Ces six catégories, traitent de la relation au temps qui passe, du vécu de stigmatisation, du besoin de maîtrise de soi, d'autrui, de son environnement, de l'expérience de la défaillance de l'environnement, de la difficile alliance avec autrui et la société, de leur situation subie tout autant que choisie de vivre en retrait du monde.

La manière dont a été constituée une des six catégories finales, nommée « Exposition à un environnement défaillant » est présentée ci-dessous. Le point de départ en sera un extrait d'entretien de recherche, réalisé avec Léon.

Dans le passage suivant, Léon rappelle le trauma qu'il a subi à l'âge de 5 ans : il est en voiture avec sa mère, son beau-père, son frère et sa sœur aînée : « Et ce dimanche-là, on l'a ramené et en fait la voiture bah, c'est sur un croisement elle est restée heu...bloquée, y’a un camion qu'est arrivé d'l'autre côté quoi, à fond... dans un virage pratiquement un virage quoi, la voiture elle est restée bloquée au milieu par heu... j'ai vu ma mère éjectée, j'ai... j’ai eu quelque chose de hein ! J'ai eu quelque chose à la jambe, j'ai eu... j'suis resté à l'hosto pendant un mois, un mois et demi ».

Ce passage d'entretien a été codé, c'est-à-dire reformulé, en restant très descriptif. Un titre lui a été adjoint au descriptif afin de faciliter le repérage de ce passage :

« La vision de la mère occulte toutes les autres souffrances ».

1. Logiciel qui permet de collecter, stocker, organiser et analyser des données issues d'enregistrements audio, vidéo... 
« Il raconte les circonstances de l'accident et rappelle encore l'image persistante de la mère éjectée de la voiture avant que d'aborder sa propre hospitalisation, assez longue, pour des problèmes à la jambe. Il ne semble pas savoir ce qu'il a lui-même subi, ne souhaite pas l'aborder ou bien le reste des souvenirs est occulté par celui de la mère. Son hospitalisation a dû pourtant être un moment de souffrance, puisqu'il avait mentionné, dans le premier entretien, à quel point il avait peur des piqûres, ce qui l'avait empêché de s'injecter de l'héroïne. Cette peur des piqûres n'est sans doute pas qu'une simple peur d'enfant. »

Ce codage a trouvé sa place avec d'autres codes, issus des entretiens réalisés avec Léon, qui, tous, faisaient référence à la confrontation à la mort et à la souffrance. Cette catégorie reste encore très descriptive, mais on voit émerger quelques propriétés (ici, il est question de tous les éléments de son parcours, où l'on retrouve des références à la souffrance et à la mort) et quelques dimensions (les différents codages, renvoyant à cet accident de voiture et à l'image traumatique de la mère, mais, aussi, les allusions à ses propres blessures physiques, aux soins qu'ils ont impliqués, sa crainte des seringues, sa toxicomanie).

Deux autres catégories descriptives serviront à construire, avec la catégorie « Souffrance et mort », une nouvelle catégorie plus abstraite : «Événements traumatiques précoces ». Ces deux catégories descriptives font référence, pour l'une, aux violences intrafamiliales, ayant entraîné le placement, retrouvées dans plusieurs récits ; pour l'autre, aux répétitions d'événements douloureux dans les différents parcours de vie. 
La catégorie «Événements traumatiques précoces » met en évidence des événements majeurs et douloureux éventuellement traumatogènes, recueillis dans les récits. Ils ont la particularité d'avoir eu lieu au cours de la minorité de leurs auteurs.

Parmi ces événements, on retrouve les traumas initiaux (accidents, maltraitances...), mais, aussi, les placements en institutions, qui se sont mal déroulés. Ces événements ont marqué leur mode relationnel dans laquelle la méfiance est majeure. Les répétitions, repérées dans les parcours de vie, montrent l'insistance d'éléments traumatiques non élaborés.

Cette catégorie sera, elle encore, regroupée avec d'autres de même niveau pour donner lieu à la catégorie plus générale encore qu'est la catégorie « traumatismes précoces ». Elle-même, regroupée avec une catégorie venant la compléter, donnera lieu à la catégorie finale « Exposés à un environnement défaillant ».

La catégorie est considérée comme finale, quand plus aucune dimension nouvelle n’apparaît. Les six catégories finales découvertes vont être présentées ci-dessous, de façon résumée. Elles ne peuvent l'être de manière détaillée dans le format d'un article, puisque chaque catégorie finale est une analyse discursive, ayant donné lieu à des textes de 10 à 15 pages chacun.

\section{Présentation des six catégories finales}

Ces six catégories finales ne pouvaient plus être regroupées entre elles mais elles étaient liées. Comme on a pu le voir précédemment, ces six catégories sont issues d'un regroupement de plus en plus général de codages antérieurs. Elles ont été produites sous forme discursive. Ces textes, constitués à partir de données ancrées dans les entretiens, ne sont donc pas 
interprétés à partir de théories, mais fournissent des éléments qui pourront, ensuite, permettre une mise en perspective avec des théories.

\section{- Exposés à un environnement défaillant}

Parmi les dix sujets rencontrés, sept ont traversé des événements traumatogènes. Pour quatre d'entre eux, ces traumas (un unique et accidentel, trois répétés et intentionnels) ont eu lieu au cours de leur enfance. Deux autres sujets ont subi des traumas répétés et intentionnels au cours de leur adolescence. Enfin, un sujet a subi un trauma accidentel et un trauma intentionnel à l'âge adulte. Trois autres sujets ont connu des situations bouleversantes (séparations familiales, changements de vie brusque) pour deux d'entre eux, au cours de l'enfance ; pour le dernier, au cours de l'adolescence.

Les particularités de ces événements sont l'absence de secours, les bouleversements de vie consécutifs qu'ils ont entraînés, mais, aussi, les solutions de protection proposée par la société. En effet, ces placements, hospitalisations ont été vécus comme une nouvelle violence. Les sujets ont ainsi développé une méfiance relationnelle, avec une tendance à se tenir à l'écart des autres. Nous avons pu relever la fréquence du clivage comme mécanisme défensif important, mais, aussi, des mouvements projectifs, comme des tendances phobiques, des angoisses d'agression, d'intrusion, une hypervigilance, des conduites addictives récurrentes, qui mettent en évidence la fragilité des enveloppes psychiques et leurs difficultés à filtrer, métaboliser, les éléments externes, tout comme à contenir les pulsions internes.

\section{- Une alliance sociale et humaine incertaine.}

Cette catégorie regroupe les éléments d'une défiance, chez les sujets rencontrés, à l'égard d'autrui, mais, de manière générale, à l'égard du social, pouvant aller jusqu'au sentiment de 
persécution. Les défaillances graves, qu'ils ont eu à subir de la part des adultes, ont eu, pour effet, de nier leur statut de sujet. Dans ces conditions, le pacte social et humain est perverti, voire, pour certains, rompu. Ainsi, si la rue marque une alternative au grand jeu social, elle prend, pour chacun, un sens différent.

\section{- À l'écart du monde}

Cette catégorie regroupe les éléments qui nous éclairent sur un certain retrait du monde, subi, mais, aussi, parfois, entretenu par les sujets. Cette tendance au retrait est liée à des expériences de vie douloureuses et précoces. Celles-ci les ont conduits à se méfier des autres, à s'en tenir à distance. Or, ce retrait relationnel entre en conflit avec les besoins narcissiques, affectifs auxquels répond autrui. C'est pourquoi cette tendance au retrait est parfois contrecarrée par des tentatives pour revenir au centre de l'attention d'autrui. Cette contradiction (refus d'autrui/besoin d'autrui) crée donc des tensions, parfois des symptômes. Ceux-ci s'intègrent dans un fonctionnement psychique particulier, reflété par un mode de vie précaire, leur permettant de maintenir la société à distance, tout en conservant avec elle un lien, de nature souvent conflictuel.

\section{- Maitrise de soi, de sa vie}

Les éléments de cette catégorie font appel à la notion de maîtrise (de soi, de son environnement, de sa vie). Ces éléments sont toujours associés à leur contraire, comme c'est le cas pour les dyades puissance/impuissance, dépendance/indépendance, actif/passif. Chez les sujets rencontrés, l'alternance d'un pôle à l'autre de ces couples se fait de manière extrême, sans ambivalence. Cela démontre les difficultés à distinguer les limites de soi, la forte tendance au clivage. L'utilisation de ces couples répond au besoin de se préserver 
narcissiquement et de tenter de poursuivre un processus de subjectivation maintes fois perturbé par des événements dramatiques.

\section{- La stigmatisation éprouvée}

Les sujets citent le regard négatif porté sur eux par autrui, mais, aussi, projeté par eux, le rejet qui l'accompagne, les émotions éprouvées et les moyens qu'ils trouvent ou non pour s'en défendre. Cette catégorie renforce l'idée d'un rapport à autrui conflictuel, d'une image narcissique de soi endommagée que la situation sociale ne vient que renforcer.

\section{- Réalité du temps qui passe}

Cette catégorie fait référence au rapport qu'entretiennent les sujets avec le temps. Ses altérations sont en lien avec le trauma et son rapport à la mort, mais, aussi, à un contexte de précarité sociale, qui semble entretenir un certain déni de la réalité temporelle.

\section{Structuration de l'analyse}

Analyser ces six catégories revient, à la fois, à trouver des liens, une cohérence, une logique qui les unit (mode empirique), mais, aussi, à faire des liens avec des théories existantes (mode théorique). Pour ce faire, le travail consistait à intégrer les catégories les unes après les autres de façon cohérente. L'apport de chacune d'entre elles a permis d'éclairer les précédentes, les compléter et a donné lieu à un constant remaniement de l'analyse. Enfin, celle-ci intègre la théorie qui entrera véritablement en discussion avec l'agencement des catégories. 


\section{DISCUSSION}

Les sujets n'ont pas tous vécu des traumas, au sens strict du terme. Certains ont été confrontés à des situations bouleversantes, qui ont eu, cependant, un fort impact psychique.

La discussion permettra tout d'abord de relever, dans ces situations de vie majeures et marquantes, les éléments subjectifs communs aux dix individus rencontrés. Ensuite elle mettra en évidence que, quels que soient leurs traits de personnalité, ces sujets présentent une logique commune, qui répond à une logique de survie. Enfin, elle abordera la place que prend la rue, comme espace physique et symbolique de la précarité sociale, dans cette logique psychique.

\section{Traumas psychiques et événements bouleversants}

Dans ces récits de vie, il est possible de remarquer à la fois, des situations allant de changements catastrophiques, d'événements bouleversants, à de véritables traumas. Parfois, il s'agit de trauma unique : à l'âge de 5 ans, Léon a perdu sa mère, son beau-père, son frère et sa sœur dans un accident de voiture, dont il a été le seul survivant. Albert a perdu sa femme et ses beaux-enfants dans un accident de la route. Chez d'autres, il est question de traumas répétés : Claire et Anne ont subi, pendant plusieurs années, des abus sexuels de membres de leur famille (le père de Claire, le grand-père maternel d'Anne). Vanille, Martin, Mick ont connu, quant à eux, des maltraitances de leurs proches (Vanille a subi des violences de son conjoint avec qui elle s'est installée à l'âge de 15 ans ; Martin a été exposé aux violences de son père, tout comme Mick à celles de son beau-père, dès leur prime enfance). Enfin, trois des sujets ont vécu ce que l'on peut qualifier de changements catastrophiques : Christophe a été placé en pension disciplinaire, par ses parents, à l'âge de 8 ans. Romane a dû surmonter, à 12 
ans, le départ d'un père, qui les a laissées, elle et sa mère, totalement désemparées. Quand Laury avait 11 ans, la famille a dû quitter la ferme paternelle et a constamment déménagé au rythme des tentatives commerciales infructueuses, entreprises par les parents. Ces événements ont, pour presque tous les sujets (hormis Albert, dont nous ne savons rien du parcours infantile et adolescent), la particularité d'avoir eu lieu au cours de leur minorité. Ainsi, les récits de vie qui nous ont été faits, trouvent leur origine dans des événements dramatiques. Quelle qu'en soit l'intensité, ils ont provoqué des changements irréversibles dans leur vie et apparaissent comme le premier maillon d'une chaîne traumatique : les parcours de vie sont effectivement émaillés de séparations, de ruptures, d'abandons. Ils sont dictés, imposés par autrui, soulevant un sentiment d'injustice face au monde des adultes. L'exemple type en est donné par le placement à caractère social ou psychiatrique, imposé à huit des dix sujets au cours de leur enfance, leur adolescence ou au début de l'âge adulte et qu'ils ont vécu comme un enfermement insupportable.

Dans ces bouleversements de vie, ils ont été régulièrement soumis aux défaillances des adultes, soit parce que ceux-ci se montraient impuissants à les aider, soit parce qu'ils étaient indifférents, soit parce qu'ils étaient eux-mêmes maltraitants. Ils ont donc trouvé peu de soutiens extérieurs, qui auraient pu faire résilience. Cette situation rejoint le point de vue de Roussillon (1991) qui, dans la lignée de Winnicott, considère que la situation initiale ne devient traumatique que si l'environnement n'apporte pas de réponse adéquate. L'enfant et l'adolescent, particulièrement dépendants de leur environnement, vivent plus directement encore cette absence d'aide. Si, par-dessus tout, l'environnement apporte des réponses inadéquates, c'est-à-dire contraires à ce qui est attendu, l'impact psychique sera destructeur. Baubet et coll. (2006) soulignent l'importance de la présence d'adultes disponibles, comme facteur de résilience chez l'enfant victime d'événements traumatogène. 
De plus, ces sujets ont eu le sentiment de ne pas avoir été entendus dans leurs éprouvés : après l'accident de voiture, qui a coûté la vie aux membres de sa famille, la tante maternelle de Léon a souhaité le recueillir, ce que les autorités n'ont pas accepté. Cette décision de placement en foyer a été un trauma supplémentaire pour l'enfant de cinq ans qu'il était.

Léon : « et en fait elle a voulu m'prendre donc elle a pas pu, moyen de... financier. Elle avait déjà un fils, elle avait des enfants... donc heu, le week-end j'étais chez elle, je fuguais j'allais chez elle, je, je... elle était là quoi ! Elle voulait m'prendre ! Ouais ! Elle voulait m'prendre!

Le chercheur : - mais l'ASE n'a pas voulu...

Léon : - Non. Donc j’ai été placé en foyer ».

Il ne cessera, par la suite, de fuguer, pour se réfugier chez cette tante. Ces fugues sont, alors, une réponse au sentiment de ne pas être entendu par les autorités.

Il a déjà été mentionné les conséquences d'un trauma sur le développement de la subjectivation, d'autant plus chez des sujets jeunes, dont le Moi immature n'est pas encore en capacité de les supporter et de les intégrer (Freud, 1937). Or, le déroulement défavorable du processus de subjectivation apparaît comme un facteur de vulnérabilité face à de nouvelles situations traumatogènes. La chaîne traumatique peut apparaître comme le vécu subjectif de sujets incapables de faire face psychiquement à une succession d'événements, qui ne cessent de faire effraction. Pour autant, l'incapacité des adultes à les en protéger, parfois même à les provoquer ne peut être niée. C'est au travers de cette contradiction, selon laquelle le danger vient de ceux dont on attendait du secours et de la sécurité, que les sujets vivent la société comme insupportablement défaillante. 
De ces expériences de vie précoces, qui les ont fait s'interroger sur le sens de leur existence, leur fragilité, leur impuissance, ressortent chez les sujets, des traits psychiques particuliers.

\section{Une logique psychique commune}

Ce qui va être évoqué ici ne correspond bien évidemment pas à la présentation d'un fonctionnement psychique, qui serait commun aux sujets enquêtés, mais à une logique qu'ils partagent bel et bien. Cette logique ouvre des perspectives sur la compréhension des pathologies limites et narcissiques, qui ne seront cependant pas développées ici.

À l'écoute des sujets interrogés, on peut percevoir des émergences pulsionnelles, qui échappent à leur contrôle, se manifestant hors de leur volonté, comme s'ils étaient immaîtrisables et qu'ils les subissaient. Elles s'expriment au travers d'actings out, mais, aussi, d'éléments présentés comme des « coups du destin », au travers de répétitions, de malchance...

Martin parle de son impulsivité. Elle peut s'exprimer de manière hétéro-agressive, par des bagarres, comme de manière auto-agressive par des tentatives de suicide. Il raconte l'un de ses passages à l'acte, alors qu'il avait intégré l'armée, où il rêvait de faire carrière. «j'me suis engagé, j'ai signé hein ! (...) l'engagement ça a pas marché (...) j'ai failli me jeter...j'ai failli me jeter par la fenêtre hein ! Alors ils m’ont mis P4 tout de suite hein ! » Son acte est présenté comme quelque chose d'immaîtrisable, qu'il regrette, mais face auquel il se résigne avec fatalité.

Les sujets vivent en effet ces différents actings out comme ne leur appartenant pas tout à fait, sans, pour autant, leur être totalement étrangers. C'est pourquoi ils seront qualifiés ici 
d'éléments non-Moi en Moi. Ces éléments sont assez proches de ce que Bion a conceptualisé par l'élément $\beta$ (Bion, 1962), faisant du psychisme non plus un appareil à penser, mais un appareil à évacuer ces éléments. Il s'agit donc bien d'éléments qui ne sont pas élaborés et dont les sources seraient en lien avec les traumas traversés, mais, aussi, avec leur environnement social (la précarité). Ce dernier favorise, en effet, les sentiments d'intrusion, mais également, de déferlement pulsionnel incontrôlable, en lien avec la perte des objets sociaux, qui sont autant d'enveloppes psychiques contenantes (habitat, statut social, famille...).

La présence de ces éléments non-Moi en Moi rend difficile le discernement des limites psychiques, qui révèlent une certaine perméabilité. Les sujets en situation de précarité oscillent alors constamment entre des postures opposées, passant d'un extrême à l'autre comme s'ils étaient équivalents. Ces couples dichotomiques ont également la particularité d'être omniprésents tels que le couple puissance/impuissance, dépendance/indépendance, position active/passive... Leur spécificité tient au fait qu'ils sont utilisés de manière confuse plutôt qu'ambivalente, qu'ils se confondent plutôt qu'ils se confrontent. Cette recherche soulève l'hypothèse du défaut de formation de la « barrière de contact », concept émis par Bion $(1962,1965)$ ou, encore, de l'aire transitionnelle de Winnicott (1971), notions proches, qui ont été prolongées par Anzieu (1985), avec la notion de Moi-peau. À la lecture de Bion, il est possible de trouver un mode explicatif cohérent avec les données : le défaut de fonctionnement de cette barrière de contact ne permet pas de maintenir suffisamment la distinction entre éléments psychiques internes et éléments externes, ainsi qu'entre tout couple de contraires. L'analyse du fonctionnement des sujets dans leur lien avec ces couples d'opposés, permet de comprendre qu'ils remplissent, là encore de manière indistincte, des buts semblables, au travers de deux fonctions essentielles, que sont la préservation narcissique et la lutte contre la désubjectivation. 
Ces deux fonctions répondent, plus généralement, à une recherche de maîtrise d'euxmêmes, de leur environnement, d'autrui, pour éviter un effondrement psychique qu'ils ont connu et qu'ils redoutent (Winnicott, 1969). Le choix inconscient d'un des deux opposés, tout comme le passage de l'un à l'autre, sera fonction de la capacité de ces pôles à protéger le narcissisme et la position de sujet des individus.

\section{Préservation narcissique}

Quel que soit le pôle du couple investi, l'objectif est toujours de tenter de sauvegarder le narcissisme : la revendication de la place de coupable, qui permet, par exemple, de conserver une posture active et subjective, remplit le même objectif que la place de victime sacrificielle. Cette seconde posture, en apparence passive, renvoie, en réalité, à une image de soi positive, quasi sainte, dont tout mal serait expulsé, projeté à l'extérieur. C'est pourquoi il est préférable de parler de « posture (faussement) passive ».

Les parcours de vie des sujets rencontrés jouent sur le manque de gratification perçue : les maltraitances, l'indifférence, rendent difficiles l'intégrité et l'investissement libidinal du Soi. La revendication d'indépendance et une certaine toute-puissance dans les récits montrent le besoin de trouver des sources de gratification.

Lutte contre la désubjectivation

L'utilisation des couples d'opposés par passage successif de l'un à l'autre de leurs deux pôles montre, en réalité, le besoin de retrouver, par le clivage, la maîtrise sur un objet, un événement, une situation ou autrui, c'est-à-dire de rester acteur, que ce soit dans une posture active ou dans une posture (faussement) passive. Ce besoin de maîtrise s'applique aussi aux éléments internes, envahissants et dangereux. Il s'agit, donc, d'éviter une posture 
d'impuissance, d'agonie et d'effondrement psychique, déjà vécu dans le passé ainsi qu'une posture de dépendance, qui ne laisse pas de place à l'expression de leur subjectivité.

Ces situations traumatiques et leurs conséquences correspondent à des situations paradoxales, telles que les théoriciens de la communication, issus de l'école de Palo Alto, les ont formulées (Watzlawick, Helmick Beavin, Jackson, 1967) en se fondant, entre autres, sur les travaux de Bateson. Il s'agit de traumatisme de non-sens ou intellectuel selon Nathan (1988).

Roussillon (1991) rappelle qu'il existe plusieurs formes de communication paradoxale : la double contrainte, qui consiste en deux informations, injonctions contradictoires, qui mettent, quoiqu'il fasse, le sujet en situation d'impasse ; la mystification, qui consiste à dénier à autrui ce qu'il ressent et perçoit, en le persuadant que ses perceptions sont fausses ; enfin, la disqualification, qui dénie au sujet ses pensées et ses paroles. Or, ce mode de fonctionnement paradoxal dans la communication est préjudiciable, car tout être humain, être éminemment social, a besoin de se référer à autrui pour se vivre et vivre le monde. Ceci est particulièrement vrai chez l'enfant, dont le rapport au monde et à soi se construit en prenant appui sur ce que lui renvoie l'adulte. C'est au travers de ses relations à l'adulte, que l'enfant va pouvoir identifier ses éprouvés, les nommer et, ainsi, se créer une réalité interne.

Quand il existe des écarts entre discours adulte et vécu infantile, les conséquences sont de plusieurs ordres : ces écarts peuvent conduire l'individu vers un fonctionnement pathologique, ou entamer la confiance que l'enfant présente habituellement en l'adulte, mais, surtout, ils vont perturber les processus de subjectivation. Comme le confirme Cahn (2006), ce sont bien des situations ou des modes de fonctionnement particuliers à autrui qui gênent le processus de 
subjectivation : l'absence ou la manque d'identification et de reconnaissance produit des perturbations à symboliser, se représenter, se situer vis-à-vis d'autrui, à reconnaître ses affects et ses pulsions.

Les événements de vie subis par ces sujets ont en commun l'attaque du processus de subjectivation et du narcissisme, conduisant à la perte d'espoir et de confiance dans le monde. Ceci est d'autant plus vrai que les sujets y ont été exposés précocement, non seulement par des traumas, mais, plus généralement, au travers de toutes situations dans lesquelles le monde adulte s'est révélé défaillant dans sa capacité d'entendre et de tenir compte de leurs éprouvés, c'est-à-dire défaillant dans leur capacité à les considérer comme sujet. Nous rejoignons en cela Ferenczi (1932), qui définit un événement traumatique comme celui dans lequel le vécu du sujet lui est dénié.

Il nous est alors plus aisé de comprendre le mode relationnel des sujets, émaillé de méfiance, de colère, de difficulté d'attachement. Les déceptions humaines répétées conduisent à un discours à la dimension paranoïaque/paranoïde, allant jusqu'à une construction délirante pour trois des sujets interrogés. Piera Aulagnier (1975) montre comment la haine perçue par l'enfant, notamment, au travers d'un « système éducatif » violent, deviendra le point nodal de toute son existence : ces abus de pouvoir peuvent amener l'enfant à considérer que toute loi est mauvaise et à déconsidérer ceux qui en sont les garants (c'est-à-dire ceux qui sont porteurs de la fonction paternelle). Chez les sujets que nous avons rencontrés, la confiance en autrui est entamée : autrui est menaçant, dominateur. S’il est aimé, cet amour sera, tôt ou tard, déçu. La croyance en un pacte social équitable, qui assurerait la sécurité de chacun, est perdue : la loi (et toute forme d'autorité qui la porte), reste celle du plus fort, vécue comme dictatoriale et injuste. Léon aborde cette question en racontant comment il a refusé, pendant plusieurs années, les aides de l'État : «Pendant un moment, j'prenais pas d'aide, hein ! Pas de RMI rien 
hein ! J'étais pas anarchie mais je ne voulais rien ! Je ne voulais rien d'eux qui, rien d'l'État, rien pour m'aider quoi ! »

Il s'agit de sujets qui se situent en totale opposition avec la logique du philosophe Thomas Hobbes, auteur contractualiste du XVII ${ }^{\mathrm{e}}$ siècle, qui voyait dans le contrat social, le moyen, pour l'individu, d'assurer sa sécurité et sa survie, là où l'homme à l'état de nature est un danger de mort potentiel pour son prochain (Haarscher, 1988), « parce qu'une société pour moi c'est pas ce qui se passe actuellement : laisser crever les gens dehors, faire les maraudes dans les marais, à faire ceci, à faire ça. Quand on voit des gens d'soixante-dix-huit ans qui claquent comme ça dehors complètement gelés, à... à voir tous ces gros problèmes et pis aller commencer à faire nos grandes gueules comme sait faire le français dans notre pays et des leçons de morale alors que au pied de notre porte y'a des gens qui crèvent hein ! Faut bien dire les choses comme elles sont. Heu non ! je... je... non. Je crois pas en ce système, quoi !» (Mick).

L'explication d'Aulagnier pourrait être complétée, car, au regard des données de terrain et des éléments théoriques relevés, il apparaît que le persécuteur se substitue à la fonction paternelle, tout comme le tout-pouvoir se substitue à la loi : ces sujets ne vivent donc pas sous l'égide de la loi du père, mais sont soumis à une toute-puissance dictatoriale, comme si elle avait pénétré en eux. Il s'agit donc de sujets, dont le fonctionnement psychique répond à une logique de survie, fondée sur la violence fondamentale (Bergeret 1984).

\section{La rue, lieu d'expression de la précarité sociale}

La situation de précarité sociale, quand l'espace de la rue devient espace de vie, peut apparaître comme la conséquence d'une histoire de vie défavorable. Elle apparaît comme un 
nouvel espace paradoxal : les sujets y vivent dans une contradiction constante, subissant les injonctions contraires d'une société qui leur reproche ce qu'elle produit (chômage, handicap, pauvreté, précarité...), les renvoyant aisément au sentiment d'inexistence sociale. Elle correspondrait à une situation désubjectivante en soi et qui réactualiserait, au travers de la perte massive d'objets sociaux, le manque de gratification narcissique. Comme toute situation paradoxale, elle allie non-sens et souffrances physiques comme psychiques. Notre recherche préliminaire nous a permis de montrer la dimension traumatogène de l'exclusion sociale, au sens où cette situation peut, à la fois, avoir une fonction initiatique, c'est-à-dire de mise en sens, tout comme elle peut rester absurde, sidérante et répéter un scénario traumatique de rupture et d'abandon. Pourtant, elle apparaît, dans les récits de vie, comme le seul espace de vie possible. C'est pourquoi se pose la question de la fonction qu'elle prend psychiquement pour les individus.

Comme le propose Houseman $(1986,2012)$, la situation paradoxale ne fait sens qu'à partir du moment où l'individu lui en donne. C'est le principe des rites initiatiques, dont, en réalité, le novice n'a rien à apprendre que le sens qu'il va donner à cette expérience de non-sens. L'expérience de la précarité, quand elle s'exprime dans la rue, peut se comprendre de différentes manières, mais, toujours, dans la logique de survie qui est la leur. Ces différents niveaux de compréhension ne peuvent se synthétiser entre eux, selon la règle proposée par la méthode complémentariste. Ils expriment tous un point de vue, qui ne s'oppose pas aux autres, mais les complète : ces différents niveaux se traduiront par une compréhension de ce que vivent les sujets d'un point de vue intra-psychique, intersubjectif et social.

D'un point de vue intra-psychique, certains sujets semblent tirer avantage de leur situation, dans le sens où la rue, bien que les conduisant à se confronter de nouveau à des situations comme l'abandon, l'absence de secours, leur permettrait de se revendiquer, cette fois, comme 
acteurs de leur survie. Elle apparaît, alors, comme une tentative de dégagement psychique, qui leur permet de prendre une posture antinomique à la posture de victime impuissante, objectalisée, qu'ils ont connue très tôt dans leur vie. La traumatophilie est évidemment présente dans le fait de revivre des situations similaires aux traumas, afin de tenter de les maîtriser, d'en être sujet et non objet passif. Au-delà, elle peut être considérée comme une expérience traumatolytique, c'est-à-dire qui peut être, à la différence des traumas passés, anticipée (Brette, 1988).

L'expérience de la rue servirait de mode d'assimilation de traumas anciens. Expérience de vie et expériences de la rue sont intriquées comme autant d'expériences formatrices/transformatrices, faisant passer les sujets d'un état de vulnérabilité à un état de force. Vanille, en particulier, mentionne la rue comme une expérience de transformation par un renforcement tant psychique que physique.

Vanille : « c’qui fait qu'après le caractère il est... ça m'a endurcie. On est quand même un peu costaud physiquement et mentalement. »

Le chercheur - physiquement on est plus costaud? »

Vanille : - ouais parce que des fois nous on voit pas mal de choses c'qui fait que automatiquement ça nous endurcit.(...) Et là maintenant après avoir connu entre guillemet "le monde de la rue", je sais qu'ça m’a beaucoup endurcie... ».

La rue renforce et non affaiblit, rend indépendant et non dépendant, à la différence de l'expérience traumatique, sur laquelle elle s'appuie pourtant. Ainsi, la rue peut être considérée comme une tentative de métabolisation d'éléments traumatiques. 
Colin et Duez (2008) complètent cette analyse, en montrant que les SDF investissent la rue et l'espace urbain comme un espace psychique. La rue serait un scénario faisant appel à des fantasmes individuels, qui ont un rapport avec la répétition de vécus traumatiques, où interviennent la rupture et la séparation. Chez ces individus qui ont perdu de nombreux étayages, il serait question d'une quête de nouveaux supports, trouvés dans l'environnement (personnages, éléments, qui s'y trouvent), afin de retrouver un espace de familiarité suffisant pour ne pas être totalement déstabilisés. Ces auteurs proposent de reprendre la notion de diffraction, à la suite de Freud (1901) et de Kaës (1988), qui consiste en une projection diffractive des différents aspects du Moi, c'est à dire d'une décomposition du Moi sur des scènes, des personnes, des éléments de l'environnement (éléments animés ou inanimés). La rue serait, pour les individus qui l'investissent comme lieu de vie, un espace d'éclaboussure d'éléments diffractés. Ceci permettrait au psychisme de se dépolluer d'éléments internes (on peut, là encore, penser aux éléments $\beta$ de Bion), en faisant basculer la scène intime sur l'espace publique.

D’un point de vue intersubjectif, la rue apporte certains bénéfices secondaires non négligeables, au fonctionnement psychique des sujets ; notamment, elle les tient à distance d'autrui, de la menace qu'il représente, ainsi que du risque de perte, qu'entraînent les relations d'attachement. C'est pourquoi nous postulons que la rue correspond, pour les sujets que nous avons rencontrés, non pas à un espace d'exclusion, mais à un espace de retrait relationnel, leur permettant de se protéger d'autrui.

En outre, la rue apparaît comme bien plus qu'un espace. Elle prend valeur de sujet : « ouais ! Et en même temps elle m'plaît c'te vie ! (...) la rue elle me plaît! J'sais pas pourquoi mais elle me plaît quoi ! T'sais j'me dis...en même temps, en même temps faut qu'j'm'en sorte ! et en même temps ça m'plait (...) mais en même temps, la rue elle 
m’appelle quoi, j'ai l'impression qu'elle m'appelle » (Léon). Ici, Léon parle de sa relation avec la rue comme s'il s'agissait d'une personne avec laquelle il serait dans une relation de dépendance presque passionnelle.

Les données anthropologiques de Houseman $(1986,2012)$ apportent un éclairage sur cette relation de type subjectif avec la rue : dans de nombreuses cultures, le lieu et le corps sont deux mondes réactifs l'un à l'autre. Pour Houseman, c'est la sensation corporelle, qui va rendre active, dynamique, la relation entre sujet et lieu. Celle-ci est, à coup sûr, assurée par la douleur. Pour cet auteur, par la douleur, le lieu devient un « sujet virtuel » et non plus un simple objet. C'est pourquoi de nombreuses processions rituelles font appel à des souffrances physiques, afin que les jeunes membres de la communauté acquièrent une véritable connaissance de ces lieux.

La rue possède ce pouvoir, cette place de sujet, avec laquelle les individus en grande précarité sont en lien. C'est le lieu investi psychiquement, où ils traversent des épreuves, où ils souffrent. Ils y réalisent une sorte d'initiation personnelle leur permettant d'assimiler des expériences à fort potentiel traumatique, qui ont bouleversé leur construction identitaire et subjective.

D'un point de vue social, la rue est revendiquée par les sujets comme un choix de vie et un espace de liberté. Cette notion de liberté s'oppose au vécu d'enfermement ressenti précédemment dans leur parcours de vie. Elle s'oppose aux contraintes qu'imposerait l'acceptation du contrat social. «j'voulais ma liberté, j'suis tranquille (...) j'dois rien à personne ». La rue est donc revendiquée comme un mode de vie choisi : « parce que au début j'étais obligé d'être dans la rue, mais là c'est mon choix !»(Christophe). 
Grace à ces revendications de liberté et de choix de vie, les sujets n'ont pas à souffrir du rejet, de l'abandon de la société. Ce sont eux, au contraire, qui rejettent le système.

Cette place d'acteur les protège narcissiquement : les sujets se voient survivre à une nouvelle épreuve, où ils tiennent, cette fois, un rôle actif. En devenant initiatique, la rue compense leur statut social négatif et leur permet de se placer en position de rivalité avec les classes favorisées, insérées et instruites. Enfin, elle leur permet de conserver l'illusion toute puissante d'une existence possible sans le social, et dans le refus de son pacte.

Cette position de sujet donne, alors, à leur expérience de la rue, une possibilité de transformer leur identité. Bien que négative, l'identité sociale que propose la situation de précarité, offrirait ce que l'on pourrait nommer une identité de recouvrement, libérant les individus de celle de victime impuissante et objectalisé.

\section{CONCLUSION}

Nous avons tenté de montrer que les situations, qui induisent un traumatisme psychique, ne se limitent pas uniquement à l'effraction causée par le trauma. Celui-ci est également induit par le bouleversement irréversible de l'environnement familial et social, que ces situations entraînent, l'absence de réponse de l'environnement ou son inadéquation, c'est-à-dire toute réponse ou absence de réponse, qui dénient leur subjectivité aux individus. C'est, donc, autant l'expérience d'anéantissement que celle de désubjectivation, qui produira le traumatisme psychique. Nous rejoignons, en cela, le point de vue d'un certain nombre d'auteurs, comme Anzieu, Green, Roussillon, qui soulignent que les situations paradoxales sont particulièrement traumatogènes.

Pour les sujets en situation de précarité rencontrés dans le cadre de cette recherche, le monde apparaît comme insupportablement défaillant. Ces parcours de vie permettent de 
comprendre comment la précarité sociale, quand elle devient un mode de vie s'exprimant dans l'espace public qu'est la rue, apparaît comme un moyen de se confronter à la société. En même temps, il s'agit d'une nouvelle épreuve à surmonter narcissiquement et identitairement. Enfin, la rue maintient à distance de la relation à autrui, vécue comme menaçante, et apparaît comme une tentative de métaboliser les traumas passés. La condition pour que la rue remplisse cette fonction est, cependant, que les sujets l'investissent dans une posture d'acteur. Cette posture leur permettrait, non pas de vivre la précarité comme une situation traumatique de plus, mais comme une tentative pour développer un processus de subjectivation, maintes fois mis à mal.

\section{RÉFÉRENCES}

Anzieu (Didier).-Le moi-peau, Paris, Dunod, 1985.

Aulagnier (Piera).- La violence de l'interprétation, Paris, Presses universitaires de France, 1975. Baubet (Thierry), Taieb (Olivier), Pradere (Jérome), Serre (Geneviève), Moro (Marie-Rose).Clinique des traumas psychiques dans la première enfance, dans Baubet (T.), Lachal (C.), OussRyngaert (L.), Moro (M.-R.), Bébés et traumas, Grenoble, La pensée sauvage, 2006, p. 37-65. Bergeret (Jean).- La violence fondamentale, Paris, Dunod, 1984.

Bergeret (Jean), Reid (Wilfrid).--Narcissisme et état limite, 2éd., Paris, Dunod, 1986.

Bion (Wilfred R.).- Aux sources de l'expérience, [2 e éd., 1962], Paris, Presses universitaires de France, 1991.

Bion (Wilfred R.).-Transformations. Passage de l'apprentissage à la croissance [2éd., 1965], Paris, Presses universitaires de France, 2002.

Brette (Françoise).- Le traumatisme et ses théories, Revue française de psychanalyse, 52, 6, 1988, p. 1259-1284.

Cahn (Raymond).- Origines et destins de la subjectivation, dans Richard (F.), Wainrib (S.), La subjectivation, Paris, Dunod, 2006, p. 7-18. 
Cierpka (Manfred), Grande (T.), Rudolf (G.), Von der Tann (M.), Stasch (M.), OPD Task Force.- The operationalized psychodynamic diagnostics system : clinical relevance, reliability and validity, Psychopathology, 40, 2007, p. 209-220.

Colin (Valérie), Duez (Bernard).-Exclusion de la scène collective et ruptures des appartenances, dans Furtos (J.), Les cliniques de la précarité, Paris, Masson, 2008, p. 72-82.

Crocq (Louis).- Le traumatisme psychique, histoire et concepts fondamentaux, dans Tovmassian (H.), Bentata (L.), Le traumatisme dans tous ses éclats. Clinique du traumatisme, Paris, In press, 2012, p. 19-47.

Devereux (Georges).- De l'angoisse à la méthode dans les sciences du comportement [1967], Paris, Flammarion, 1980.

Devereux (Georges).- Essais d'ethnopsychiatrie générale [1970], Paris, Gallimard, 1977.

Devereux (Georges).-Ethnopsychanalyse complémentariste [1972], Paris, Flammarion, 1985.

Fazel (Seena), Khosla (Vivek), Doll (Helen), Geddes (John).- The prevalence of mental disorders among the homeless in Western countries: Systematic review and meta-regression analysis, PLoS medicine, 5, 12, 2008, p. 1670-1680.

Ferenczi (Sandor).- Le traumatisme [1932], Paris, Payot, 2006.

Fierdepied (Sophie), Sturm (Gesine), Taïeb (Olivier), Moro (Marie-Rose), Baubet (Thierry).Aspect traumatogène de l'exclusion sociale, une analyse ethnopsychanalytique, Annales médicopsychologiques, 170, 5, 2012, p. 338-341.

Freud (Sigmund).- Le rêve et son interprétation [1901], Paris, Gallimard, 1985.

Freud (Sigmund).-Au-delà du principe de plaisir [1920], Paris, Payot, 2010.

Freud (Sigmund).-Inhibitions, symptômes et angoisses [1926], Paris, Presses universitaires de France, 2011.

Freud (Sigmund).- L'analyse avec fin et l'analyse sans fin [1937], dans Résultats, idées, problèmes II- 1921-1938, Paris, Presses universitaires de France, 1985, p. 231-268.

Freud (Sigmund).- L 'homme Moïse et la religion monothéiste [1939], Paris, Gallimard, 1986. Glaser (Barney), Strauss (Anselm).- La découverte de la théorie ancrée. Stratégie pour la recherche qualitative [1967], Paris, Armand Colin, 2010. 
Green (André).- La mère morte, dans Green (A.), Narcissisme de vie, narcissisme de mort, Paris, Les éditions de minuit, 1983, p. 222-253.

Green (André).- Genèse et situation des états limites, dans André (J.), Les états limites, Paris, Presses universitaires de France 1999, p. 23-68.

Haarscher (Guy).--La raison du plus fort, philosophie du politique, Liège-Bruxelles, Margada, 1988.

Houseman (Mickaël).- Note sur quelques propriétés générales de la transformation initiatique, métamorphoses de l'identité. Initiations et sectes, Nouvelle revue d'ethnopsychiatrie, 6, 1986, p. $31-40$.

Houseman (Mickaël).- Le rouge est le noir. Essais sur le rituel, Toulouse, Presse universitaire du Mirail, 2012.

Kaës (René).- La diffraction des groupes internes, Revue de psychothérapie psychanalytique de groupe, 11, 1988, p. 169-174.

Kovess-Masfety (Viviane).- Précarité et santé mentale, Paris, Doin, 2001.

Lacan (Jacques).- Le temps logique et l'assertion de certitude anticipée, dans Écrits, Paris, Presses universitaires de France, 1966, p. 197-213.

Laporte (Anne), Chauvin (Pierre).- La santé mentale et les addictions chez les personnes sans logement personnel d'Ile-de-France, Rapport final, Observatoire du SAMU social de Paris, 2010 [le 24 mars 2015,

http://www.inserm.fr/content/download/8550/63979/version/1/file/samenta.pdf]

Lebigot (François).- Le traumatisme psychique, Bruxelles, Fabert, 2001.

Nathan (Tobie).--Le sperme du diable, Paris, Presses universitaires de France, 1988.

OPD Task force.- Operationalized psychodynamic diagnosis OPD-2, Manual of diagnosis and reatment planning, Cambridge, MA, Hogrefe and Huber, 2008.

Paille (Pierre), Mucchielli (Alex).-L'analyse qualitative en sciences humaines et sociales, $3^{\mathrm{e}}$

éd., Paris, Armand Colin, 2012.

Roussillon (René).- Paradoxes et situations limites de la psychanalyse, Paris, Presses universitaires de France, 1991.

Roussillon (René).-Agonie, clivage et symbolisation, Paris, Presses universitaires de France, 1999. 
Roussillon (René).- Pluralité de l'appropriation subjective, dans Richard (F.), Wainrib (S.), La subjectivation, Paris, Dunod, 2006. p. 59-80.

Schiltz (Lony), Houbre (Barbara), Martiny (Caroline).- Précarité sociale, marginalisation et pathologie limite : étude comparative de plusieurs groupes de sujets en rupture de projet de vie, L'évolution psychiatrique, 72, 3, 2007, p. 453-467.

Strauss (Anselm), Corbin (Juliet).-Basics of qualitative research [3 $3^{\mathrm{e}}$ éd.,1990], Newbury park, CA, Sage, 2008.

Sturm (Gesine), Nadig (Maya), Moro (Marie Rose).- Writing therapies. An ethnographic approach to transcultural therapies, Forum qualitative social research, 11, 3 art. 1, 2010, [1e 24 mars 2015, http://www.qualitative-research.net/index.php/fqs/article/view/1558/3071] Wainrib (Steven).- Un changement de paradigme pour une psychanalyse diversifiée, dans Richard (F.), Wainrib (S.), La subjectivation, Paris, Dunod, 2006, p. 19-57.

Watzlawick (Paul), Helmick Beavin (Janet), Jackson (Don De Avilla).- Une logique de la communication [1967], Paris, Seuil, 1972.

Winnicott (Donald Woods).- La crainte de l'effondrement et autres situations cliniques [1969], Paris, Gallimard, 1975.

Winnicott (Donald Woods).- Jeu et réalité [1971], Paris, Gallimard, 1975. 